angesichts der sich sehr schnell verändernden politischen Rahmenbedingungen, kurz: Kalter Krieg, auch als wenig realistisch. ${ }^{181}$

\title{
4.2 Die lange Vorgeschichte des Monat
}

Der Verlauf der 13 Nürnberger Prozesse fiel in eine Phase, in der sich der Charakter der Deutschlandpolitik der amerikanischen Besatzungsbehörde mehr und mehr veränderte. Die sich bereits während des Zweiten Weltkrieges in der US-amerikanischen Administration herauskristallisierenden rivalisierenden konzeptionellen Vorstellungen hinsichtlich der Frage, wie nach dem militärischen Sieg über das »Hitler-Regime« mit dem »deutschen Problem« umzugehen sei, entfalteten sich im Laufe der Zeit und standen nicht zuletzt in einem unmittelbaren Zusammenhang mit dem sich nach der Potsdamer Konferenz sukzessive anbahnenden Ost-West-Konflikt. Wie gezeigt, verloren unter Truman und seinen antikommunistisch eingestellten Beratern die Anhänger des sogenannten Morgenthauplanes, die sich für eine harte Bestrafung Deutschlands aussprachen, gegenüber den Verfechtern des War Department und des State Department, die eine »weiche Linie « favorisierten, an politischem Einfluss. ${ }^{182}$ Infolgedessen galt es nicht mehr, drei der zentralen Punkte des Potsdamer Abkommens, d.h. demilitarization, denazificazion und decartelization, umzusetzen. Fortan war die Sowjetunion der »natürliche« Feind, sodass spätestens zwei Jahre nach dem Ende des Krieges das primäre außenpolitische Ziel der Truman-Regierung darin bestand, Deutschland zum Bündnispartner zu machen und das Land in das Netzwerk internationaler Institutionen einzubinden und vermittels Kooperationen zu zähmen.

Gleichwohl stand am Anfang die konkrete amerikanische Besatzungspolitik in Deutschland noch unmittelbar im Zeichen der Direktive JCS 1067, die bekanntlich die miteinander verknüpften Ziele des Bestrafens und der Entnazifizierung der deutschen Gesellschaft sowie eine umfassende Demokratisierungspolitik beinhaltete. ${ }^{183}$ Obwohl es aufseiten der Vereinigten Staaten kein eindeutiges konzeptionelles Programm gab, stand eines fest: Nach dem Zusammenbruch des >Dritten Reiches< sollte Deutschland kein weiteres Mal die Gelegenheit bekommen, den internationalen Frieden zu gefährden, sodass Militarismus und Nazismus in jedweder Form ausgerottet werden sollten. Daraus folgte auch, dass sämtliche Institutionen und Denkweisen der NS-Diktatur beseitigt werden sollten, damit eine grundsätzliche Veränderung der deutschen politischen Kultur in Gang gebracht werden konnte, was freilich einen radikalen Bruch sämtlicher Traditionen zur Folge haben sollte, die das »Hitler-Regime« und seine Verbrechen erst möglich machten. Hiermit sollten Voraussetzungen geschaffen werden,

181 Siehe Reichel, Vergangenheitsbewältigung in Deutschland, S. 71.

182 Siehe die entsprechenden Ausführungen in Kap. I.2.4.

183 Zur Konzeption der US-amerikanischen Besatzungsmacht im Hinblick auf die »Transformation zur Demokratie« Westdeutschlands vgl. aus der neueren Forschungsliteratur besonders die beeindruckende, anhand von umfangreichen originalen Direktiven, Handbüchern und Technischen Anweisungen nachgezeichnete und einer soziologischen Analyse unterzogene Studie von Uta Gerhardt, Soziologie der Stunde Null. Zur Cesellschaftskonzeption des amerikanischen Besatzungsregimes 1944-1945/1946, Frankfurt a. M. 2005. 
um Deutschland die Chance zu geben, in den Kreis der zivilisierten Völker zurückzukehren. ${ }^{184}$

Mithin stand in der amerikanischen Besatzungszone - wie auch in der britischen und französischen - der politische Wiederaufbau Deutschlands im Zeichen der Herstellung von demokratischen Zuständen. So besaß die Zulassung politischer Parteien neben den auf Kreis- und Landesebene eingesetzten Bürgermeistern, Landräten und Ministerpräsidenten - einen großen Stellenwert. Abgesehen von neonazistischen und offen »rechten « Parteien hatte dies zur Folge, dass neben den vier großen Richtungen KPD, SPD, CDU und Liberale - auch zahlreiche kleine Parteien sowie Regionalparteien gegründet werden konnten. Hiermit sollte der eigentliche Rahmen abgesteckt werden, auf dem sich das parteipolitische Leben entwickeln und früher oder später die Bevölkerung ihre demokratischen Rechte in Anspruch nehmen konnte. Bereits im Jahre 1946 erlaubten die Amerikaner Wahlen zu verfassungsgebenden Landesversammlungen, und im selben Jahr, spätestens aber 1947, konnte die Bevölkerung ihre ersten Landtage wählen. ${ }^{185}$

Weitaus schwieriger gestaltete sich der Versuch, im Rahmen des Demokratisierungsprogramms die in Potsdam beschlossene Entnazifizierung durchzuführen. ${ }^{186}$ Während die getroffenen Maßnahmen in der sowjetischen Besatzungszone im Kontext eines intendierten grundlegenden Systemwechsels unter »antifaschistisch-demokratischen « Vorzeichen durchgeführt wurden, ${ }^{187}$ sah man sie in den westlichen Zonen als entscheidende Vorbedingung einer moralischen Rehabilitierung Deutschlands und der Veränderung der politischen Kultur an. ${ }^{188}$ In der Anfangsphase war die Entnazifizierungspolitik dadurch charakterisiert, neben der Auflösung der NSStrukturen, der Internierung von führenden NS-Funktionären sowie der Verhaftung von Kriegsverbrechern, ehemalige Nazis aus dem gesamten öffentlichen Leben und der Wirtschaft zu entfernen. Vor allem in der amerikanischen Zone wurde unter General Clay die politische Säuberung rigoros durchgesetzt. Schätzungsweise 200000 Personen wurden in den Westzonen gemäß Kategorie »automatic arrest« in Internierungslager verbracht, wobei sich diese Zahl bereits um die Jahreswende 1946/47 um die Hälfte reduziert hatte. In Haft blieb der Personenkreis, der in dem vom Nürnberger Militärtribunal als »verbrecherische Organisation« eingestuften Institutionen (SS, einschließlich SD und Gestapo sowie dem Korps der Politischen Leiter der NSDAP) einen Rang bekleidet hatte. Zudem wurden noch innerhalb des ersten Jahres der Besatzungszeit etwa 150000 Personen aus dem öffentlichen Dienst sowie rund 70000 Personen aus der Wirtschaft von ihren Positionen entbunden.

184 Siehe Konrad Jarausch, Die Umkehr. Deutsche Wandlungen 1945-1995, Bonn 2004, S. 31 f.

185 Vgl. Benz, Potsdam 1945, S. 135 ff.

186 Vgl. grundsätzlich Clemens Vollnhals (Hg.), Entnazifizierung. Politische Säuberung und Rehabilitierung in den vier Besatzungszonen 1945-1949, München 1991.

187 Vgl. grundsätzlich Helga Welsh, »Antifaschistisch-demokratische Umwälzung« und politische Säuberung in der sowjetischen Besatzungszone Deutschlands, in: Klaus-Dietmar Henke/Hans Woller (Hg.), Politische Säuberung in Europa. Die Abrechnung mit Faschismus und Kollaboration nach dem Zweiten Weltkrieg, München 1991, S. 84-107, sowie Wolfgang Meinicke, Zur Entnazifizierung in der sowjetischen Besatzungszone unter Berücksichtigung von Aspekten politischer und sozialer Veränderung 1945-1948, Diss. Berlin (Ost) 1983.

188 Das Folgende nach Reichel, Vergangenheitsbewältigung in Deutschland, S. 30-37; Jarausch, Die Umkehr, S. 66-75, sowie Benz, Potsdam 1945, S. 157-182. 
Mit dem Militärgesetz Nr. 8 erhielt die Entnazifizierung Ende September 1945 insofern eine neue Dimension, weil hiermit die Möglichkeit geschaffen wurde, massiver in die Wirtschaft einzugreifen. Gleichwohl wurde auch immer mehr der bürokratische Charakter sichtbar, der neben den bereits genannten Gründen mitverantwortlich war, dass schließlich die Mehrheit der deutschen Bevölkerung von ihrer anfänglichen Zustimmung für Entnazifizierungsmaßnahmen mehr und mehr abrückte. Die USMilitärregierung (OMGUS) versuchte nämlich zuerst mittels des äußerst umstrittenen Fragebogens, der insgesamt 131 separate Fragen umfasste, Personen in Schlüsselfunktionen vor ihrer Wiedereinstellung zu überprüfen. Ab Januar 1946 wurde dann durch die Kontrollratsdirektive Nr. 24 für die zu entlassenen Personen eine neue Grundlage geschaffen, die auf Vereinheitlichung abzielte. Durch die Festlegung von 99 Kategorien für Nationalsozialisten und Personen, die von den Alliierten verdächtigt wurden, ihnen feindlich gegenüber zu stehen, konnten Personen ohne jede tatsächliche individuelle Prüfung entlassen werden. Die ablehnende Haltung der deutschen Bevölkerung und noch mehr das schematische Vorgehen und die Überdehnung der Entnazifizierungsdirektiven, die zum einen für Verwirrung sorgte und zum anderen den Wiederaufbau des öffentlichen Dienstes und der Wirtschaft beeinträchtigte, bildeten die Voraussetzungen dafür, dass die Amerikaner sich entschieden, die Deutschen am Verfahren zu beteiligen.

Durch das »Gesetz zur Befreiung von Nationalsozialismus und Militarismus« vom 5. März 1946 und die Einführung des "Spruchkammerverfahrens « bezogen die Amerikaner deutsche Stellen in die Durchführung der Entnazifizierung ein. Das Befreiungsgesetz stellte den Versuch dar, zwischen dem Diskriminierungs- und Strafgedanken sowie der als notwendig erachteten Einzellfallprüfung und der gerichtlichen Ermessungsentscheidung einen Ausgleich zu schaffen. Gleichwohl zeigte sich alsbald, dass die Einführung des rechtsstaatlichen Verfahrens mit hohen politischen Kosten verbunden war. Denn der immense bürokratisch-gerichtliche Aufwand der umfassenden politischen Säuberung und das tatsächliche Ergebnis klafften zusehends auseinander. Was als radikale Entnazifizierung der deutschen Gesellschaft konzipiert wurde, mit der alle ehemaligen Nazis aus dem öffentlichen Leben entfernt und gegebenenfalls bestraft werden sollten, führte zu einer umfassenden Rehabilitierungsmaßnahme.

Nach dem Befreiungsgesetz musste jeder Deutsche, der älter als 18 Jahre alt war, einen Fragebogen ausfüllen, der nach fünf formalen Belastungskategorien unterschied und über den in den Spruchkammern, einem Laiengericht bestehend aus einem Vorsitzenden und zwei ehrenamtlichen Richtern, entschieden wurde. Diese Kategorien waren: I. Hauptschuldige (primär die Angehörigen der vom Internationalen Militärtribunal als verbrecherisch verurteilten Organisationen: SS, Gestapo, Korps der politischen Leiter der NSDAP); II. Belastete (Aktivisten, Militaristen, Nutznießer); III. Minderbelastete (Bewährungsgruppe); IV. Mitläufer; V. Entlastete. Allein in der amerikanischen Zone mussten knapp 13 Millionen Menschen den Fragebogen ausfüllen. Hierbei war knapp ein Drittel vom Befreiungsgesetz betroffen, wobei am Ende nur etwa zehn Prozent von einer Spruchkammer tatsächlich verurteilt wurden und hier wiederum nur eine Minderheit mit tatsächlichen Strafen oder Nachteilen von Dauer Leben mussten. Entlastung brachte im August 1946 die Jugendamnestie für alle nach 1919 Geborenen und die Weihnachtsamnestie 1946, die Kriegsgeschädigte und sozial Schwächere begünstigte. Trotz der großen Nachsicht der Spruchkammerpraxis war das Verfahren durchaus umstritten. Vor allem die dem traditionellen Strafrecht 
widersprechende Verfahrenspraxis, wonach die Betroffenen die Beweislast für ihre Unschuld zu erbringen hatten, gab Anlass zur Kritik, weil dies zur Folge hatte, dass erstens in zahlreichen vergleichbaren Fällen völlig verschiedene Entscheidungen getroffen werden konnten, zweitens sich eine »Persilschein-Praxis« durchsetzen konnte, weil sich die Mitglieder der ehemaligen »Volksgemeinschaft« gegenseitig Unbedenklichkeitsbescheinigungen über ihre Vergangenheit ausstellen konnten, und drittens sowohl Fragebogenfälschungen und Begünstigungen als auch eine Denunziationspraxis Tür und Tor geöffnet wurde; weniger aus politischer Überzeugung als aus Gründen wirtschaftlicher Konkurrenz bzw. persönlicher Feindschaft. Zudem sorgte nicht zuletzt der schleppende Gang der Spruchkammerpraxis für Zündstoff, die Vielzahl von eher harmlosen Fällen gegenüber den »schweren« vorrangig zu behandeln. Hierdurch entstand in der Bevölkerung die Ansicht: >Die Kleinen hängt man, und die Großen lässt man laufen.< Dies führte dazu, dass die unmittelbar nach Kriegsende anzutreffende Zustimmung von 57 Prozent in der Bevölkerung für die Entnazifizierungspolitik zu dem Zeitpunkt am Sinken war, als mit dem Inkrafttreten des Befreiungsgesetzes sich das Verfahren auf die gesamte Gesellschaft ausdehnte, sodass aufgrund der überzogenen Ziele und der bürokratischen Durchführung am Ende nur noch 17 Prozent eine zustimmende Haltung einnahmen. Angesichts dieser Umstände und nicht zuletzt aufgrund des sich abzeichnenden Ost-West-Konfliktes erlahmte spätestens im Frühjahr 1948 aufseiten der Amerikaner das Interesse an der Entnazifizierung. Nachdem die Sowjetunion in ihrer Zone die Entnazifizierung im März beendete, entschlossen sich auch die Amerikaner, das Verfahren zu beenden. ${ }^{189}$

Gleichwohl ist zu berücksichtigen, dass bereits am Anfang der Entnazifizierung das Verfahren öffentlich unter Beschuss geriet. Hierbei waren es nicht nur ehemalige Nazis, deren Ablehnung keineswegs überraschen konnte, sondern bereits im Frühjahr 1946 kritisierte der Rat der Evangelischen Kirche in einem offenem Brief an die USMilitärregierung, dass das Befreiungsgesetz >elementare Rechtsgrundsätze< verletze und für neues Unrecht sorge. Unter den Skeptikern und Kritikern befanden sich selbst ehemalige Gegner der NS-Diktatur, die die politische Säuberung grundsätzlich befürworteten, aber nicht zuletzt die tatsächliche Spruchkammerpraxis ablehnten. Beispielsweise plädierte der ehemalige Häftling des Konzentrationslagers Buchenwald und Mitherausgeber der Zeitschrift Frankfurter Hefte Eugen Kogon ausdrücklich für das Recht auf politischen Irrtum, und der Präses der Hessischen Landkirche, Martin Niemöller, ebenfalls KZ-Häftling, rief sogar im Laufe des Jahres 1948 öffentlich zum Boykott der Entnazifizierung auf. ${ }^{190}$

Von besonderer Bedeutung war die Entnazifizierung im Kultur- und Geistesleben. Angesichts des großen Stellenwertes, die die Erneuerung der politischen Kultur im Rahmen der alliierten Nachkriegskonzepte spielte, war freilich die personelle Überprüfung unabdingbar. Der Versuch der »Umerziehung« der Deutschen setzte auf einen radikalen kulturellen Neuanfang, der eine grundlegende Entnazifizierung und

189 Vgl. insgesamt Klaus-Dietmar Henke, Die Trennung vom Nationalsozialismus. Selbstzerstörung, politische Säuberung, »Entnazifizierung«, Strafverfolgung, in: Ders./Woller, Politische Säuberung in Europa, S. 21-83.

190 Reichel, Vergangenheitsbewältigung in Deutschland, S. 35. Zur Kritik seitens der evangelischen Kirche an der Entnazifizierung vgl. insgesamt Clemens Vollnhals, Evangelische Kirche und Entnazifizierung 1945-1949. Die Last der nationalsozialistischen Vergangenheit, München 1989. 
Kontrolle der kulturellen Sphäre voraussetzte. ${ }^{191}$ Insbesondere für die Besatzungsbehörden der US-Zone und in der sowjetischen Zone arbeiteten zahlreiche renommierte sogenannte Kulturoffiziere, die mit der Geschichte der deutschen Philosophie, Literatur, Kunst und Musik bestens vertraut waren. Darüber hinaus wurden sie von international bekannten deutschen und österreichischen Intellektuellen - die in vielen Fällen jüdischer Herkunft waren - beraten, die vom Nationalsozialismus zur Emigration gezwungen wurden und im Zuge des Krieges mit den Armeen ihrer Aufnahmeländer oder zu einem späteren Zeitpunkt als Remigranten zurückkehrten. Beispielsweise fungierte in der US-Zone Hans Habe als Kulturoffizier, der insbesondere in der Anfangsphase einen großen Einfluss im Hinblick auf die Pressepolitik besaß.

Inmitten der materiellen Not in der unmittelbaren Nachkriegszeit entwickelte sich in der deutschen Bevölkerung ein großes Bedürfnis nach Kultur. Deshalb war den Alliierten die Wiederbelebung des kulturellen Lebens wichtig, weil man im Kontext der Erziehung der Deutschen zur Demokratie gleichzeitig sozusagen Propaganda für die eigene Kultur und mithin für das eigene politische System machen konnte. Hierbei waren in der Anfangsphase die inhaltlichen Unterschiede in der alliierten Kulturpolitik nur schwer auszumachen, was sich durch die Übergangssituation erklären ließ und erst durch den Ausbruch des Kalten Krieges zu klar erkenntlichen und divergierenden Konzepten führte. Am schärfsten wurde die Entnazifizierung in der sowjetischen Besatzungszone und vor allem in der US-Zone durchgeführt, wo schätzungsweise 600 Personen in Schlüsselpositionen im Kultursektor (Verlagswesen, Theater, Film, Presse und Rundfunk) aufgrund ihrer politischen Vergangenheit eine Beschäftigung untersagt wurde. Allerdings bezog sich die Entnazifizierung nicht nur auf Personen. Da auch die "geistigen Wurzeln« des Nationalsozialismus ausgemerzt werden sollten, wurden sämtliche Druckerzeugnisse hinsichtlich ihrer Inhalte überprüft; beispielsweise führte die Überprüfung der Bibliotheken dazu, dass noch Mitte 1946 knapp 15000 Bücher und 150 Zeitschriften auf dem Index standen und der Öffentlichkeit vorenthalten wurden.

Trotz alledem entwickelte sich das kulturelle Leben in den vier Zonen in der Anfangsphase im Grunde genommen ziemlich frei. Dass es in der sowjetischen Besatzungszone um den Aufbau einer sozialistischen Kultur nach sowjetischen Vorgaben gehen sollte, zeichnete sich 1945/46 nicht mit Deutlichkeit ab. Auch hier wurde zu Beginn bewusst ein facettenreiches Spektrum von geistigen und künstlerischen Strömungen zugelassen. Zahlreiche Künstler und Intellektuelle zeigten sich gegenüber der sowjetischen Kulturpolitik durchaus aufgeschlossen, weil in diesen Kreisen die Forderung nach einer Auseinandersetzung mit der Geschichte des >Dritten Reiches nicht selten mit der Kritik am Kapitalismus - den sie für das Aufkommen des Nationalsozialismus mitverantwortlich machten - und der Hoffnung nach einem alternativen Wirtschaftssystem korrespondierte. In diesem Zusammenhang schien ihnen die sowjetische Kulturpolitik mit dem anvisierten Konzept der »Stunde Null« insgesamt attraktiv, weil nämlich hierdurch nicht zuletzt die Möglichkeit zu bestehen schien, die radikale Neugestaltung sowohl der gesellschaftlichen als auch der wirtschaftlichen Verhältnisse in Deutschland zu erreichen - was sich in vielen Fällen explizit und implizit mit dem Wunsch nach einer sozialistischen Utopie verband. Ein nicht zu unter-

191 Das Folgende nach Axel Schildt, Kultur und geistiges Leben, in: Benz, Deutschland unter alliierter Besatzung, S. 134-140, hier S. $134 \mathrm{f}$. 
schätzender Vorteil der Kulturbürokratie der Sowjetischen Militäradministration in Deutschland (SMAD) sollte darin bestehen, dass sie das ideologisch-politische Modell des Sozialismus den deutschen Intellektuellen in einer Form präsentierten, die erstaunlich liberale und pragmatische Züge aufwies, ohne dass sich der stalinistische Hintergrund zeigte. ${ }^{192}$

Nach Ansicht des Tübinger Historikers Michael Hochgeschwender war das Problembewusstsein der Sowjets im Hinblick auf die nationalsozialistische Vergangenheit gut ausgebildet, sodass in ihrem Sektor unter der Ägide von SMAD und der KPD ein quasi antifaschistisch-»demokratischer« Grundkonsens entstand, der zur »politischkulturellen Ratio« der Ostzone (und später der DDR) werden sollte. In diesem Zusammenhang führte er weiter aus:

Der antifaschistische Konsens mit seinem spezifischen Demokratieverständnis wurde bald mit zwei weiteren wichtigen Komponenten verknüpft. Zum einen ging es um die alte Forderung nach der Einheit der Arbeiterbewegung, wodurch unmittelbar die Sozialdemokratie zum Objekt stalinistischer Interessen wurde, zum anderen fand seit 1946 eine konsequente Adaption des nationalen Gedankens von Seiten der deutschen Kommunisten und der SMAD statt. Indem diese vier strategischen Ziele (sozialistische Demokratie, Antifaschismus, Einheit der Arbeiterbewegung und nationale Einheit) auf eine Weise propagiert wurden, in der genuin stalinistische Töne vorerst nur zurückhaltend eingesetzt wurden, wandten die Sowjets erneut jene Taktik an, die zu Zeiten Willi Münzenbergs so erfolgreich gewesen war. Cerade das strikte Festhalten am Antifaschismus der Kriegszeit führte dazu, daß ein nicht unerheblicher Teil der linksintellektuellen Emigranten in der Ostzone ihre politische Heimat fand. ${ }^{193}$

In diesem Kontext avancierte der am 3. Juli 1945 gegründete »Kulturbund zur demokratischen Erneuerung Deutschlands« zu einer entscheidenden Einrichtung. Mit Sitz in Berlin fungierte er quasi als organisatorisches Zentrum für den Versuch, vor dem Hintergrund der »Ausrottung der faschistischen Ideologie« zur »demokratischen« Erneuerung Deutschlands unter antifaschistischen Vorzeichen insbesondere allen literarischen und künstlerisch Interessierten ein politisch unverdächtiges Forum zu bieten. Auf breite Resonanz stieß der Kulturbund unter anderem dadurch, dass im Mittelpunkt des Programms die Forderung stand, >die große deutsche Kultur, den Stolz unseres Vaterlandes, wieder erwecken und ein neues deutsches Geistesleben begründen zu wollen. ${ }^{194}$ Hiermit kam ausdrücklich zum Ausdruck, worin das Hauptanliegen des Kulturbundes lag, nämlich einen wesentlichen Beitrag bei der »kulturell-moralisch-geistigen Vereinigung « Deutschlands ${ }^{195} \mathrm{zu}$ leisten, die freilich auf antifaschistisch-»demokratischer« Basis erfolgen sollte.

Die Organisation wurde in der Anfangsphase noch von allen vier Besatzungsmächten gefördert und auch in den drei Westzonen auf lokaler Ebene zugelassen. Seine

192 Vgl. Wolfgang Schivelbusch, Vor dem Vorhang. Das geistige Berlin 1945-1948, Frankfurt a. M. 1997, S. $55-58$.

193 Hochgeschwender, Freiheit in der Offensive?, S. $130 \mathrm{f}$.

194 Anne Hartmann/Wolfram Eggeling, Sowjetische Präsenz im kulturellen Leben der SBZ und frühen DDR 1945-1953, Berlin 1998, S. 189.

195 Schivelbusch, Vor dem Vorhang, S. 119. 
Hauptaktivitäten entfalte der Kulturbund in Berlin. Als Präsident des Kulturbundes fungierte der kommunistische Schriftsteller Johannes R. Becher, dessen zentrales Ziel darin bestand, mit dem Kulturbund Künstler und Intellektuelle aus dem bürgerlichliberalen, dem sozialdemokratischen und auch dem katholischen Lager anzusprechen und an sich $\mathrm{zu}$ binden. Ebenfalls sollten Emigranten gewonnen werden sowie apolitische Künstler und Intellektuelle, die während der NS-Diktatur im »inneren Exil« lebten. Beim Kulturbund handelte es sich um eine "fellow traveller«-Bewegung, die zweifelsohne in der konzeptionellen Tradition Willi Münzenbergs stand, sodass die verdeckten kommunistischen Absichten der Initiatoren nur »Experten« ersichtlich werden konnten, die gewissermaßen Erfahrungen mit den berühmten Agitations- und Propagandatechniken der Kommunisten hatten. ${ }^{196}$ In diesem Zusammenhang wurde alsbald auch versucht, prominente Künstler für den eigenen Sektor zu gewinnen, obwohl sie - wie zum Beispiel Wilhelm Furtwängler oder Gustaf Gründgens - politisch belastet waren. Vor dem Hintergrund des auf den ersten Blick nicht klar erkennbaren Führungsanspruches der KPD/SED zeigte sich die Führung des Kulturbundes bei der inhaltlichen Ausrichtung überaus flexibel und besetzte Themen, die bei einem breitem Publikum auf Interesse stießen und bis 1947 auch für den zonenübergreifenden Erfolg verantwortlich waren. Mitverantwortlich dafür war sicherlich, dass der Kulturbund hochrangige Persönlichkeiten an sich binden konnte - sie reichten von Schriftstellern wie Ludwig Renn, Ricarda Huch, Anna Seghers, Günther Weisenborn oder Willi Bredel, dem Romanisten Viktor Klemperer, dem Philosophen Ernst Niekisch bis zu den »nützlichen Idioten« wie beispielsweise dem SPD-Politiker Gustav Dahrendorf oder dem CDU-Politiker Ferdinand Friedensburg. So gelang es dem Kulturbund, nach nur einem Jahr 45000 Mitglieder für sich zu gewinnen, die sich in 394 Ortsgruppen organisierten. Bis zum Jahr 1948 konnte die Zahl der regulären Mitglieder auf 120000 gesteigert werden. ${ }^{197}$

Als zentrales Thema wurde ab September 1946 die Einheit des deutschen Nationalstaates besetzt und mit den sowohl zeitlich als auch inhaltlich überschneidenden Neutralitäts- und Friedenskampagnen verzahnt. Nachdem die SED und die SMAD anfingen, immer mehr gegen die sich abzeichnenden Pläne seitens der Briten und Amerikaner zu polemisieren, die planten, ihre beiden Zonen zusammenzulegen (Bizone), und sich für den Erhalt eines zentralistisch verfassten deutschen Nationalstaates aussprachen und die Kulturpolitik in der SBZ insgesamt nach direkten sowjetischen Vor-

196 Willi Münzenberg organisierte bekanntlich den berühmten »Ersten Internationalen Kongreß der Schriftsteller für die Verteidigung der Kultur«, der im Juni 1935 in Paris stattfand und der durch die Volksfrontstrategie geprägt war. Entsprechend der seit 1934 von Stalin verkündeten neuen Linie, wonach nicht mehr die kommunistische Revolution auf der Tagesordnung stand, sondern in Namen des Antifaschismus die europäischen kulturellen Werte - für die vor allem der sowjetische Humanismus stehen sollte-, gegen den deutschen Nationalsozialismus zu verteidigen, wurden französische und internationale antifaschistische Intellektuelle mobilisiert. Primär ging es aber darum, der Kommunistischen Partei in Frankreich durch das Gewinnen von bürgerlichen Intellektuellen im Wahlkampf zu helfen. Eingeladen wurden u. a. André Gide, André Malraux, Louis Aragon, Heinrich Mann, Johannes R. Becher, Bertold Brecht, Ilja Ehrenburg, Aleksei Tolstoi und Aldous Huxley. Schivelbusch (Vor dem Vorhang, S. 123) weist darauf hin, dass Becher hier bei der Vorbereitung und Durchführung des Kongresses seine ersten praktischen Erfahrungen gemacht hat, die ihm in dann später beim Kulturbund nützlich waren.

197 Hermann, Kultur im Wiederaufbau, S. 104. 
gaben erfolgte sowie sich verstärkt abgrenzenden Tendenzen zuwandte, veränderte sich mithin auch der Charakter des Kulturbundes. Im Verlauf des Jahres 1947/48 begannen zahlreiche Intellektuelle aus dem bürgerlichen und katholischen Milieu, den Kulturbund zu verlassen. Angesichts der militanten antiamerikanischen Propaganda seitens führender Exponenten des Kulturbundes kam es im November $1947 \mathrm{zu}$ einem Verbot in der amerikanischen und britischen Besatzungszone in Berlin. ${ }^{198}$

Die Absicht des Kulturbundes, eine »demokratische« Erneuerung unter antifaschistischen Vorzeichen in Deutschland zu erreichen, fand seinen Niederschlag in der von ihr herausgegebenen »kulturpolitischen Monatszeitschrift« mit dem Titel Aufbau, die unter sowjetischer Lizenz Ende September 1945 erstmalig erschien. In der bewusst überparteilich konzipierten Zeitschrift veröffentlichten in den ersten Heften nicht nur die Herausgeber Heinrich Mann, Theodor Plivier, George Lukács, Ferdinand Friedensburg und Ernst Wiechert. Beispielsweise schrieben auch Hans Fallada und Thomas Mann Aufsätze für ebendieses Organ. ${ }^{199}$

Die französische, britische und amerikanische Besatzungspolitik zeichnete sich in der ersten Phase, die bis Ende 1947/Anfang 1948 reichte, dadurch aus, dass die rigide Entnazifizierung mit umfassenden Demokratisierungsmaßnahmen koinzidierte. »Re-Education« bzw. »Umerziehung« der Deutschen lautete das anspruchsvolle und ambitionierte Programm, mit dem der autoritären gesellschaftlichen und ideengeschichtlichen Tradition in Deutschland demokratische Werte entgegengesetzt werden sollten, was freilich die Auseinandersetzung mit dem Nationalsozialismus sowie den deutschen Massenverbrechen beinhaltete. Der Schwerpunkt der Umerziehungsbemühungen lag in den Westzonen hierbei bei den Amerikanern. Im Hinblick auf das Ziel der Etablierung einer grundsätzlich demokratischen politischen Kultur in Deutschland wurde im Kontext der Umerziehungspolitik neben dem Bildungswesen und Rundfunk dem gesamten Medienbereich eine große Bedeutung beigemessen, wobei in diesem Zusammenhang nicht zuletzt die (Tages- und Wochen-)Presse und die kulturpolitischen Zeitschriften eine besondere Rolle spielen sollten. Die Verantwortung für das gesamte Mediensystem lag zunächst in den Händen der »Psychological Warfare Division«, die unter der Kontrolle der alliierten Streitkräfte stand und von dem US-amerikanischen Kulturoffizier Hans Habe geleitet wurde und die seitens der Amerikaner nach der Errichtung der Besatzungsadministration umgewandelt wurde in »Information Control Division« (später »Information Services Division«). Hier wurde über die schriftliche Zulassung von Zeitungen, Magazinen, Zeitschriften, Büchern, Broschüren und sonstigen Veröffentlichungen entschieden, die von Deutschen herausgegeben werden sollten. Denn publizistische Äußerungen jedweder Art durch Deutsche waren von einer Lizenz abhängig. Die Vergabepraxis von Lizenzen unterschied sich in der Anfangsphase in der französischen Zone gegenüber der britischen und amerikanischen Zone kaum, und erst in der nachträglichen Kontrolle der einmal vergebenen Lizenz zeigten sich Letztere weniger rigide. Denn vor dem Hintergrund der Verpflichtung der sogenannten Lizenzträger, bestimmte Inhalte, in erster Linie nationalsozialistischer Art, nicht zu veröffentlichten und zudem keine Kritik an der konkreten Politik der Besatzungsmächte zu formulieren, zeigten sich Engländer und Amerikaner liberaler und duldeten beispielsweise in deutschen Zeitungen ihrer

198 Siehe Hochgeschwender, Freiheit in der Offensive?, S. 135-137.

199 Benz, Potsdam 1945, S. 196. 
Zonen - zumindest in der Anfangsphase - auch zum Teil Kritik gegenüber den Maßnahmen ihrer Besatzungsbehörden. ${ }^{200}$

Im Hinblick auf die Tageszeitungen besaß in der unmittelbaren Nachkriegszeit vor allem die von der amerikanischen Militärregierung in München herausgegebene Neue Zeitung einen hohen Stellenwert. Sie avancierte zur bedeutendsten deutschsprachigen Zeitung der ersten Besatzungsjahre. ${ }^{201}$ Vom Oktober 1945 an erschien diese Tageszeitung als offizielles Organ der amerikanischen Besatzungsmacht zehn Jahre lang für die deutsche Bevölkerung. Die Neue Zeitung sollte einen entscheidenden Beitrag leisten bei der »Amerikanisierung«bzw. Demokratisierung Deutschlands und im Kontext der Umerziehung der Gesellschaft, die Besiegten dazu zu bringen, ihre Schuld ohne Vorbehalt auf sich zu nehmen. Mithin kreisten die Themen um die "großen« Fragen der Schuld, der verlorenen Werte, der Sprache der Macht, über die Eigenheiten und Sonderwege der deutschen Geschichte, über das geistige Klima sowie alsbald über die zunehmend schärfere Teilung des Landes. Unter der anfänglichen Leitung des Chefredakteurs und US-Majors Hans Habe (von April 1946 übernahm Hans Wallenberg dieses Amt und trat es im September $1947 \mathrm{ab}$ ), der sich unter anderem auf die Mitarbeit von dem in die USA emigrierten Romancier Stefan Heym verlassen konnte, veröffentlichten im Laufe der Zeit hochrangige Philosophen, Schriftsteller, Politiker und politische Publizisten: zum Beispiel Heinrich und Thomas Mann, Alexander Mitscherlich, Hermann Hesse, Alfred Andersch, Heinrich Böll, Eugen Kogon, Hermann Kesten, Max Horkheimer, Theodor W. Adorno, Kurt Schumacher oder Theodor Heuss. Bei einer Startauflage von 500000 Exemplaren - zeitweise betrug die Auflage 2,5 Millionen Exemplare ${ }^{202}$ und erreichte rund 10 Millionen Leser - besaß, verglichen mit den anderen Ressorts, das Feuilleton eine besondere Funktion. Unter der Leitung von Erich Kästner öffnete das Feuilleton der deutschen Bevölkerung das »Fenster zur literarischen Welt«, das während der NS-Diktatur verschlossen war, und leistete somit inmitten der "Trümmerlandschaft « auf diesem Weg einen nicht zu unterschätzenden Beitrag beim Ziel der »Verwestlichung« der Deutschen. Es erschienen beispielsweise Texte von internationalen Schriftstellern und Philosophen wie T. S. Eliot, Paul Claudel, Jean-Paul Sartre, Ignazio Silone, Julien Green, Ernest Hemingway oder auch von Ortega y Gasset. ${ }^{203}$ Im Feuilleton ging die Neue Zeitung auch auf die Entnazifizierungspolitik nicht zuletzt in der amerikanischen Besatzungszone ein und würdigte sie in der Anfangsphase als notwendige Maßnahme. ${ }^{204}$ Zudem setzte man sich intensiv mit dem Nationalsozialismus auseinander: Neben der Thematisierung der Nürnberger Prozesse schaltete man sich beispielsweise in die "große« Diskussion über die Streit-

200 Ingrid Laurien, Politisch-kulturelle Zeitschriften in den Westzonen 1945-1949. Ein Beitrag zur politischen Kultur der Nachkriegszeit, Frankfurt a. M. 1991, S. 36.

201 So Helmuth Mosberg, REeducation. Umerziehung und Lizenzpresse im Nachkriegsdeutschland, München 1991, S. 43.

202 Hans Habe, Im Jahre Null, München 1977, S. 108.

203 Vgl. Wilfried F. Schoeller (Hg.), Diese merkwürdige Zeit. Leben nach der Stunde Null. Ein Textbuch aus der »Neuen Zeitung«, Frankfurt a. M. 2005.

204 Bereits in seinem Geleitwort zur ersten Ausgabe der Neuen Zeitung am 18. Oktober 1945 hob Eisenhower als Oberbefehlshaber der amerikanischen Truppen in Europa und als Militärgouverneur der amerikanischen Besatzungszonen in Deutschland ausdrücklich hervor, dass das Organ auch die Aufgabe hat, der deutschen Bevölkerung die Notwendigkeit der Entnazifizierungspolitik zu vermitteln. Das Geleitwort ist abgedruckt in: Ebd., S. $15 \mathrm{f}$. 
schrift Jaspers ein - in der sich der deutsche Philosoph bekanntlich gegen die These von einer Kollektivschuld des deutschen Volkes für die vom »Hitler-Regime« begangenen Massenverbrechen wandte - und initiierte eine eigenständige differenzierte und anspruchsvolle Debatte über die deutsche Schuld, an der sich auf Ersuchen der Redaktion auch Jaspers selbst beteiligte. ${ }^{205}$ Im Zusammenhang mit der »Vergangenheitsbewältigung« ging die Neue Zeitung zudem ausdrücklich sowohl auf den nationalsozialistischen Antisemitismus als auch auf den alsbald nach Kriegsende in Erscheinung tretenden neuen Antisemitismus ein. In einem am 16. August 1946 veröffentlichten Artikel vertrat Habe den Standpunkt, dass die Rehabilitierung Deutschlands, die Rückkehr Deutschlands in den Kreis der westlichen Kulturnationen, davon abhinge, wie die Deutschen das Zusammenleben mit den Juden politisch regeln und inwiefern der Antisemitismus institutionell und gesetzlich gebannt werde. Vor dem Hintergrund seines Appells an die deutsche Regierung, deutsche Juden aus der Emigration zurückzurufen, plädierte Habe für die Versöhnung der Deutschen mit den Juden und befürwortete angesichts der begangenen Verbrechen eine Wiedergutmachung, die für ihn ausschließlich eine deutsche und nicht eine Angelegenheit der Alliierten sein musste. Der Chefredakteur der Neuen Zeitung sprach sich indes gegen eine formelle und oberflächliche Wiedergutmachung aus und forderte stattdessen, dass die Deutschen ein ehrenhaftes Bemühen - auch materiell - zeigen müssten, um die Versöhnung mit den Juden auch nach außen hin unter Beweis zu stellen. Denn, so führte Habe aus: »Die Welt muß den Wunsch Deutschlands fühlen, das begangene Unrecht wenigstens an den[nen] gutzumachen, die das Unrecht überlebt haben. $\aleph^{206}$

In Anbetracht seiner Auffassung, dass die »Behandlung der Judenfrage« einerseits zu einem Symbol der Menschlichkeit geworden und ein Barometer für die "politische Reife eines Volkes« und andererseits der Antisemitismus in Deutschland zu einem »Symbol der deutschen Unverbesserlichkeit« zu betrachten sei, beklagte die Neue Zeitung die mangelnde Bereitschaft einer breiten Mehrheit der deutschen Bevölkerung zur direkten Versöhnung mit den Juden. ${ }^{207}$

Zweifelsohne besaßen die Neue Zeitung und andere Tageszeitungen (Frankfurter Rundschau, Die Welt etc.) sowie Wochenzeitungen (u. a. Die Zeit) im Rahmen der Umerziehungspolitik in den westlichen Zonen eine große Bedeutung. Gleichwohl kann festgestellt, dass mit Blick auf sämtliche Presseerzeugnisse in diesem Zusammenhang die Gründung einer großen Anzahl allgemeiner politisch-kultureller Zeitschriften einen wichtigeren Beitrag leisten sollten. Sie besaßen vor allem aufgrund ihrer konzeptionellen Anlage eine wichtigere Funktion bei der Konstituierung einer grundsätzlich demokratischen politischen Kultur bzw. eines demokratisch-parlamentarischen System im Nachkriegsdeutschland. Bevor dies anhand der im Oktober 1948 von der amerikanischen Militärregierung gegründeten Zeitschrift Der Monat genauer beschrieben und erklärt werden wird, soll im Folgenden in aller Kürze ein Blick auf drei unmittelbar nach Kriegsende ins Leben gerufene politisch-kulturelle Zeitschriften geworfen werden, die sowohl aus personeller als auch aus inhaltlicher Sicht durchaus

205 Siehe Elisabeth Matz, Die Zeitungen der US-Armee für die deutsche Bevölkerung (1944-1946), Münster 1969, S. 75-96.

206 Zit. n. Kim Kyong-Kun, Die Neue Zeitung im Dienste der Reeducation für die Deutsche Bevölkerung 1945-1946, Phil. Diss. München 1974, S. 177.

207 Ebd., S. 175 u. $177 \mathrm{f}$. 
zur unmittelbaren »Vorgeschichte« des Monat gehören. Hierbei geht es um Die Wandlung, Der Ruf und die Frankfurter Hefte. Sie stehen exemplarisch für die große Zahl von politisch-kulturellen Zeitschriften, die insbesondere in den ersten Nachkriegsjahren grundsätzlich einen großen Anteil an der Konstituierung demokratischen Denkens in den westlichen Zonen Deutschlands hatten; nicht zuletzt aufgrund ihres pluralistischen Charakters. Bei allen Unterschieden in der inhaltlichen Grundausrichtung spielte die Auseinandersetzung mit dem Nationalsozialismus und der Entnazifizierungspolitik in den westlichen Zonen in den drei Zeitschriften in unterschiedlichem Maße eine Rolle.

Im Spätherbst 1945 gründete in der US-Zone der zukünftige Herausgeber Dolf Sternberger eine der ersten Zeitschriften in der "Stunde Null« mit dem programmatischen Titel Die Wandlung. ${ }^{208}$ Während ihres Bestehens bis zum Jahre 1949 fungierten Karl Jaspers, Alfred Weber und Werner Krauss als Mitherausgeber. Angesichts des Erscheinungsortes Heidelberg und der wissenschaftlichen Tradition der Herausgeber knüpfte man an die Weimarer Zeit an, sodass "philosophische und soziologische Konstellationsanalysen einen breiten Raum« einnahmen. ${ }^{209}$ Zum Mitarbeiterkreis zählten unter anderem emigrierte Freunde und Schüler von Weber und Jaspers wie zum Beispiel die 1933 aus Deutschland vertriebene jüdische Philosophin Hannah Arendt. Daneben veröffentlichten zeitgenössische Schriftsteller wie Hans-Egon Holthusen, Marie Luise Kaschnitz, Erich Kästner und der jüdische Historiker und Nationalismusforscher Hans Kohn. Trotz ihres eher akademischen Habitus wandte sich die Zeitschrift auch aktuellen Themen der Politik zu, die nicht zuletzt durch Carlo Schmidt, Adolf Arndt und Heinrich von Brentano erörtert wurden. Das wesentliche Ziel bestand darin, nach der nationalsozialistischen Barbarei einen Beitrag zu leisten, damit sich in Deutschland ein »moderner Humanismus« (Sternberger) durchsetzen konnte. Vor dem Hintergrund der »deutschen Schuld« spielte auch die Auseinandersetzung mit der Geschichte des >Dritten Reiches` eine wichtige Rolle. Von herausragender Bedeutung waren in diesem Zusammenhang sicherlich die beiden Aufsätze Organisierte Schuld und Konzentrationsläger von Arendt, ${ }^{210}$ die im Kern bereits ganz wesentliche Elemente ihrer Interpretation des Nationalsozialismus als ein System "totaler Herrschaft « enthielten und in ihre Totalitarismusstudie einflossen. ${ }^{211}$ Hierbei ging es unter anderem in der »Stunde Null« darum, sowohl über die deutschen Massenverbrechen aufzuklären als auch die nationalsozialistische Ideologie in allen Poren der Gesellschaft zu beseitigen. So erschien zudem in der Wandlung von 1945 bis 1948 eine Artikelserie, in der die drei Autoren Dolf Sternberger, Gerhard Storz und Wilhelm E. Süskind die Sprache und Semantik des Nationalsozialismus enthüllten, die erstaunliche Ähnlichkeit mit Viktor Klemperers 1947 erschienenen Lingua Tertii Imperii (LTI) aufwies und später selbst als Buch erschien. ${ }^{212}$

208 Vgl. Monika Waldmüller, Die Wandlung, Marbach a. N. 1988.

209 Harry Pross, Literatur und Politik. Ceschichte und Programme der politisch-literarischen Zeitschriften im deutschen Sprachgebiet seit 1870, Freiburg i. Br. 1963, S. 136

210 Hannah Arendt, Organisierte Schuld, in: Die Wandlung 1 (1946), H. 4, S. 333-344, sowie dies., Konzentrationsläger, in: Die Wandlung 3 (1948), H. 4, S. 309-330.

211 Arendt, The Origins of Totalitarianism.

212 Dolf Sternberger/Gerhard Storz/Wilhelm E. Süskind, Aus dem Wörterbuch des Unmenschen, Hamburg 1957; Victor Klemperer, LTI. Notizbuch eines Philologen, Berlin 1947. 
Allerdings kamen in der Wandlung auch Personen zu Wort, die eine unverhohlene Kritik an den Entnazifizierungsmaßnahmen zum Ausdruck brachten. So bemängelte Karl Heinrich Knappstein in seinem Beitrag Die versäumte Revolution, dass die Entnazifizierung keine Revolution der Deutschen selbst sei, mit der ehemalige Nazis aus führenden Positionen entfernt würden, sondern als eine administrative Maßnahme der alliierten Sieger durchgeführt wurde. ${ }^{213}$ Und Rudolf Krämer-Badoni kritisierte in seiner Veröffentlichung Terror der Anständigen in scharfen Tönen, dass von den Entnazifizierungsmaßnahmen auch die vielen »kleinen « Mitglieder der NSDAP betroffen waren, obwohl sie eigentlich - so wie der Autor - nur »Mitläufer« gewesen wären:

Durch die schematische Gesetzgebung sind wir Zwangsmitläufer zu schuldigen Mitläufern geworden. Wir sind in die schiefe Stellung von Verdächtigen geraten. Mit einem Mal gehörten wir dazu, ob wir uns innerlich als Gegner betrachteten oder nicht. Dem Geiste des Gesetzes folgend, müssen wir froh sein, wenn wir uns von dem Verdacht reinwaschen können. Wir müssen froh sein, wenn wir mit der Etikette `Nur unmaßgeblich nazistisch davongekommen. Wie sollen wir nun noch Antinazis sein können, ohne schon wieder in den Verdacht von Opportunisten zu geraten. ${ }^{214}$

Mit einer zeitweise erreichten Auflage von 120000 Exemplaren avancierte die in München mit einer amerikanischen Lizenz ausgestattete Zeitschrift Der Ruf zu einer der meistgelesenen und einflussreichsten Blätter der ersten Nachkriegsjahre. ${ }^{215}$ Nachdem sie im Winter 1944/45 noch als »Kriegsgefangenen-Zeitung« von einem Personenkreis herausgegeben wurden, der sich in amerikanischen Lagern befand, übernahm im Herbst 1945 ein neues Redaktionsteam dieses Blatt. Chefredakteur wurde Walter Mannzen, der mit seinem führenden Mitarbeiter Hans Werner Richter alsbald mit den amerikanischen Besatzungsbehörden in Schwierigkeiten geriet. Der Hauptgrund hierfür war die zum Teil einseitige Kritik an der »Kollektivschuld«, an den umfassenden Umerziehungsmaßnahmen sowie an dem Nürnberger Hauptkriegsverbrecherprozess; Letzteren lehnten beide ab und forderten stattdessen »revolutionäre deutsche Gerichte ${ }^{216}$

Von Sommer 1946 bis Mitte des Jahres 1947 erschien Der Ruf für zehn Monate lang unter der Leitung der beiden Herausgeber Alfred Andersch und Hans Werner Richter. In der Zeitschrift, die mit dem Untertitel Unabhängige Blätter der jungen Generation erschien, sollte vor allem die »junge heimkehrende Generation« zu Worte kommen, die die Hoffnung hatte, einen radikalen Neuanfang zu starten. Hierbei handelte es sich um eine Gruppe engagierter Vertreter der Zwischengeneration im Alter zwischen 30 und 40 Jahren, die ihre ersten politischen Erfahrungen in der Zeit der Weimarer Republik gemacht hatten, sich zum Teil als politisch aktive Antifaschisten verstanden und nach Hitlers »Machtergreifung« ins Exil gingen; zum Beispiel waren Andersch und Richter in diesen Jahren aktive Kommunisten, und Andersch war für ein halbes

213 Karl Heinrich Knappstein, Die versäumte Revolution, in: Die Wandlung 2 (1947), H. 8, S. 663-677.

214 Rudolf Krämer-Badoni, Terror der Anständigen, in: Die Wandlung 2 (1947), H. 5, S. 379-391.

215 Vgl. grundsätzlich Jérôme Vaillant, Der Ruf. Unabhängige Blätter der jungen Ceneration (19451949). Eine Zeitschrift zwischen Illusion und Anpassung, München u. a. 1978.

216 Hans Schwab-Felisch, Einleitung, in: Ders. (Hg.), Der Ruf. Eine deutsche Nachkriegszeitschrift, München 1962, S. 10-18, hier S. 11. 
Jahr in einem nationalsozialistischen Konzentrationslager inhaftiert. Die beiden Herausgeber versammelten im Ruf nicht zuletzt eine »heimatlose Linke«, die allerdings als undogmatische Sozialisten bzw. Marxisten keineswegs blind waren gegenüber den tatsächlichen Verhältnissen in der Sowjetunion unter Stalin und in der sowjetischen Besatzungszone. Das gemeinsame Band der Autoren - zu ihnen zählten unter anderem Arthur Koestler, Karl Krolow, Hans Schwab-Felisch, Wolfdietrich Schnurre, Hans Sahl - bestand vor allem in dem Wunsch nach einem freien sozialistischen Europa. Vor dem Hintergrund der Enttäuschung mit dem "östlichen Kollektivismus« implizierte dies einen Weg über den »Sozialismus mit einem menschlichen Gesicht«, der am Ende bei einem »sozialistischen oder radikaldemokratischen Humanismus« landen sollte, der sich von den "politischen Machenschaften aller vier Besatzungsmächte zu distanzieren versuchte $\ll{ }^{217}$ Angesichts der politischen Erfahrungen mit dem Nationalsozialismus und dem Stalinismus erschien ebendieser Sozialismus notwendig, um zukünftig »den Frieden« in der Welt zu sichern, und aus diesem Grund sollte die Teilung Deutschlands in einen östlichen sowie einen westlichen Teil verhindert werden. Für Andersch erschien eine `freie deutsche Republik« als zentrales politisches Ziel, die er als eine »ideale Pufferzone « zwischen dem westlichen und östlichen Gesellschaftssystem betrachtete. Die Vorstellung eines unabhängigen Deutschlands zwischen den beiden Blöcken erschien den drei Westmächten als problematisch und politisch durchaus gefährlich, sodass die der »Truman-Doktrin« und zusehends einem antikommunistischen Kurs« folgende US-amerikanische Militärverwaltung, die sich mithin die Integration »West-Deutschlands« in das eigene westliche System zum Ziel gesetzt hatten, den Rufzu einem Herausgeberwechsel zwang. ${ }^{218}$ Nach dem 16. Heft wurde Der Ruf verboten. Obwohl die genauen Umstände nicht eindeutig geklärt sind, spielte hierbei eine Rolle, dass die Zeitschrift im Verdacht stand, einen »wiederauferstandenen Nationalismus« zu vertreten. Dieser Verdacht wurde sehr frühzeitig erhoben, aufgrund der artikulierten politischen Forderung nach einer Vereinigung Deutschlands, um eine »Haltbarkeit des Friedens« zu erzielen. Er mischte sich mit dem Misstrauen der Amerikaner, die in der Zeitschrift immer mehr einen speziellen >Nihilismus $\mathbf{z u}$ entdecken glaubten. ${ }^{219}$

Nach der Aufhebung des Verbotes wurde die Zeitschrift übergangsweise unter der stellvertretenden Schriftleitung von Erich Kuby weitergeführt. Mit der Veröffentlichung der ersten Ausgabe des Jahres 1948 wurde Der Ruf von Walter von Cube neu herausgegeben, konnte aber nicht mehr an die politische Bedeutung und Wirkung der zurückliegenden Zeit anknüpfen. Nachdem die Zeitschrift im Laufe des Jahres 1949 bedeutungslos geworden war, stellte sie ihr Erscheinen ein.

Ein Grundzug des Rufs war die nicht nachlassende, teilweise vehemente Kritik an der Umerziehungspolitik und am Entnazifizierungsverfahren vor allem der Amerikaner. Vor dem Hintergrund der frühzeitig artikulierten Kritik am Nürnberger Militärtribunal stand die Zeitschrift den Entnazifizierungsmaßnahmen grundsätzlich kritisch gegenüber, obwohl die politische Ausschaltung von Nationalsozialisten aus

217 Hermann, Kulturim Wiederaufbau, S. 62.

218 Ebd., S. 62.

219 Vgl. Schwab-Felisch, Einleitung, hier S. $14 \mathrm{ff}$. 
öffentlichen Ämtern als legitim und notwendig betrachtet wurde. ${ }^{220}$ Aber durch den umfassenden Charakter würden sie den Geist des Nationalsozialismus atmen. ${ }^{221}$ Letzten Endes sah die Zeitschrift sogar die Gefahr, dass der Versuch der Entnazifizierung der deutschen Gesellschaft nicht nur ein denkbar unvollkommenes Instrument zur Bekämpfung des Nationalsozialismus darstellen würde, sondern sowohl die individuelle Auseinandersetzung über die persönliche Schuld verhindere als auch das Ressentiment in der Bevölkerung zum Kochen bringen lasse. So schrieb Kuby: »Mit Sorge sieht man, wie durch die Arbeit der Spruchkammern ohne andere Schuld der Beteiligten als die, daß sie den Apparat der Denazifizierung bedienen, die Reste des Rechtsgefühls im Volke zerstört werden und an seine Stelle eine neue Form dynamischen ,Volksempfindens< tritt. «222

Infolgedessen konnte es nicht verwundern, dass Der Ruf sich ganz besonders für die Jugendamnestie einsetzte. Hierbei scheute die Redaktion sich nicht, einen ehemaligen jungen Offizier der Waffen-SS das Wort ergreifen zu lassen. ${ }^{23}$

Gewisse Gemeinsamkeiten mit der Ausrichtung des Rufs existierten mit der 1946 von Eugen Kogon und Walter Dierks (mit Clemens Münster und W. M. Guggenheimer) gegründeten Monatszeitschrift Frankfurter Hefte. Auch hier trat man angesichts eines anvisierten radikalen politisch-moralischen Neubeginns neben dem Engagement für ein demokratisches Europa mit Blick auf den Neuaufbau Deutschlands für einen »demokratischen Sozialismus« ein, der indes auf einem christlichen bzw. linkskatholischen Fundament gebaut werden sollte. ${ }^{224}$ Hierbei handelte es sich um einen »wirtschaftlichen Sozialismus « auf einer demokratischen Grundlage, was unter anderem die Überführung der Großindustrien und Banken in Gemeineigentum implizierte. Zentraler Gesichtspunkt war hierbei keineswegs eine verstärkte Macht des Staates, sondern das Befürworten einer »Markt-Planwirtschaft des freien Sozialismus«, der eine Mischform aus kapitalistischen und sozialistischen Elementen darstellen sollte. Höchstes Ziel war eine »Demokratie des wirtschaftlichen, sozialen und kulturellen Wohlstandes «, die aufgrund ihrer in Freiheit erreichten Vergesellschaftung auch den besten Schutz "gegen jeden Totalitarismus« biete. ${ }^{225}$

Auch in den Frankfurter Heften wurde frühzeitig Kritik formuliert am Nürnberger Hauptkriegsverbrecherprozess ${ }^{226}$ und dem Entnazifizierungsverfahren. So vertrat beispielsweise Kogon im Jahre 1947 in seinem Beitrag Das Recht auf politischen Irrtum die Auffassung, dass angesichts der angestrebten Ziele das gesamte Entnazifizierungsverfahren als verfehlt zu betrachten sei und zudem mit katastrophalen gesellschaftlichen Folgen koinzidiere. Auch die in der US-Zone erfolgte Jugend- und Weihnachts-

220 Siehe [Red. Der Ruf,] Irrealistische Säuberung. Zur Reform des Befreiungsgesetzes, in: Der Ruf 2 (1947), H. 19, S. 5 f.

221 E[rich] K[ub]Y, Denazifizierung der Demokraten, in: Der Ruf 2 (1947), H. 11, S. 3.

222 Ders., Die Geburt der Schuld, in: Der Ruf 2 (1947), H. 23, S. 1.

223 R. A., »Gebt uns eine Chance!«, in: Der Ruf 2 (1947), H. 23, S. 7.

224 Vgl. exemplarisch Walter Dierks, Das Wort Sozialismus, in: Frankfurter Hefte 1 (1946), H. 7, S. 628643, sowie K[arl] H[einrich] Knappstein, Die Stunde der Sozialreform, in: Ebd., H. 3, S. 1-3.

225 Eugen Kogon, Der Weg zu einem Sozialismus der Freiheit in Deutschland, in: Frankfurter Hefte 2 (1947), H. 9, S. 877-896.

226 Siehe exemplarisch Eugen Kogon, Gericht und Gewissen, in: Frankfurter Hefte 1 (1946), H. 1, S. 25-37, sowie Walter Dierks, Nürnberg und die Geschichte, in: Ebd., H. 1, S. 35. 
amnestie hätte am wesentlichen Charakter nichts geändert, weil immer noch »fünf Millionen und mehr Menschen « allein von den britischen und amerikanischen Entnazifizierungsmaßnahmen praktisch betroffen seien, da sie infolge der Spruchkammerpraxis aus dem Berufsleben ausgeschlossen seien. ${ }^{227}$

Seine Skepsis gegenüber der Entnazifizierung kam in diesem Zusammenhang bei der politisch aufgeladenen Diskussion um die sogenannten kleinen Mitläufer und deren Rolle beim Wiederaufbau Deutschlands zum Ausdruck. Für ihn war dieser Personenkreis nicht automatisch moralisch kompromittiert, sodass sie nicht von vornherein von der Teilnahme am demokratischen Prozess ausgeschlossen werden könnten. Kogon sprach sich für eine konkrete Modifizierung des Entnazifizierungsverfahrens aus, die auf einer notwendigen Unterscheidung zwischen Schuld und Irrtum beruhen sollte:

Wir wollen es ohne Umschweife aussprechen: Es ist nicht Schuld, sich politisch geirrt zu haben. Verbrechen zu verüben oder an ihnen teilzunehmen, wäre es auch nur durch Duldung, ist Schuld. Und Fahrlässigkeit ist ebenfalls Schuld, wenn auch eine von anderer und geringerer Art als Verbrechen und Verbrechenteilnahme. Aber politischer Irrtum - in allen Schattierungen - samt dem echten Fehlentschluß gehört weder vor Gericht noch vor Spruchkammern. ${ }^{228}$

Die in unterschiedlichem Maße in den drei Zeitschriften Die Wandlung, Der Ruf und Frankfurter Hefte - die hier stellvertretend für viele andere stehen - zum Ausdruck gebrachte Skepsis und Kritik am Nürnberger Militärtribunal und an den Entnazifizierungsmaßnahmen, die sich explizit und implizit in erster Linie an die amerikanische Besatzungsmacht richtete, zählte zu den allgemeinen Hintergründen für die Gründung des Monat. Genauso wie die Tatsache, dass sich in der deutschen Bevölkerung nach einer anfänglichen breiten Zustimmung für den Hauptkriegsverbrecherprozess, im Zusammenhang mit den zwölf Nachfolgeprozessen und den Entnazifizierungsmaßnahmen immer mehr eine ablehnende Haltung breitmachte, die nicht zufällig mit einer Ablehnung einer angeblichen deutschen »Kollektivschuld« angesichts der nationalsozialistischen Verbrechen korrespondierte und gewissermaßen in eine »Opfermentalität« mündete. Ebendiese Aspekte müssen mitbedacht werden im Hinblick auf die Gründe, die dazu führten, dass die amerikanische Militärregierung sich dazu entschloss, den Monat ins Leben zu rufen. Allerdings spielte das grundsätzliche politische Verhältnis der Vereinigten Staaten als unbestrittene Führungsmacht der westlichen Länder und der Sowjetunion in diesem Zusammenhang eine entscheidendere Rolle. Denn angesichts der allgemeinen Entwicklung der internationalen Beziehungen zwischen den beiden einzig verbliebenen Supermächten, in deren Mittelpunkt auch und vor allem die »Deutschlandfrage«stand, mussten sich die Vorgänge im Laufe des Jahres 1947 (Zusammenlegung der amerikanischen und britischen Zone zur »Bizone« und somit Herausbildung eines westdeutschen Kernstaates, Verkündung der Truman-Doktrin und des Marshallplanes, Kominform-Gründung im September und Verkündung der »Zwei-Lager-Theorie« durch Schdanow im Auftrage Stalins) auch auf die speziellen kulturpolitischen Aktivitäten der beiden Staaten auswirken. Mit anderen

227 Eugen auf politischen Irrtum, in: Frankfurter Hefte 2 (1947), H. 7, S. 641-655 (Zitat S. 646).

228 Ebd., S. 649 f. 
Worten: Die Entwicklung der konkreten Deutschlandpolitik der Vereinigten Staaten und der Sowjetunion stand fortan ausdrücklich im Zeichen des Ost-West-Konfliktes. In diesem Zusammenhang sollten nicht zuletzt die deutschen Intellektuellen eine besondere Rolle spielen.

Bereits der erste gesamtdeutsche Schriftstellerkongress in Berlin vom 4 . bis 8 . Oktober 1947 im noch nicht gänzlich geteilten Berlin stand bereits deutlich im Zeichen der Lagerbildung im Nachkriegsdeutschland. ${ }^{22}$ Obwohl er auf Einladung des Schutzverbandes Deutscher Autoren (SDA) stattfand, war der eigentliche Initiator der Kulturbund zur demokratischen Erneuerung Deutschlands, der hierbei von der Kulturabteilung der Sowjetischen Militäradministration (SMAD) sowohl finanziell als auch organisatorisch unterstützt wurde. Am letzten Tag des Kongresses untersagten die Amerikaner dem Kulturbund, den sie seit längerer Zeit für kommunistisch dominiert hielten, jede Tätigkeit in ihrem Sektor; im November kam es - wie gesagt schließlich zu seinem Verbot in den amerikanischen und britischen Sektoren Berlins. Infolgedessen konnte es auch nicht überraschen, dass es im Verlauf des Kongresses zu erheblichen Spannungen kam. Mehr als 280 Schriftsteller, Kritiker, Verleger sowie Berichterstatter aus den vier Besatzungszonen und aus aller Welt folgten dem Aufruf von Kulturbund und SDA; hier trafen auch zum ersten Male zahlreiche Exilanten mit Vertretern der »inneren Emigration« zusammen. Zum Beispiel gehörten Ricarda Huch, Elisabeth Langgässer, Anna Seghers, Axel Eggebrecht, Alfred Kantorowicz, Stefan Hermlin und Hans Mayer zu den Teilnehmern. Ausdrücklich nicht eingeladen wurden Autoren, die während der Zeit der NS-Diktatur Mitglied der NSDAP waren oder als Schriftsteller und Journalist einen Beitrag geleistet hatten bei der Verbreitung des »nationalsozialistischen Geistes«. Zu den wenigen ausländischen Gästen gehörte neben drei Schriftstellern aus der Sowjetunion ein damals noch unbekannter amerikanischer Journalist. Der Präsident des Kulturbundes, Johannes R. Becher, beabsichtigte auf dem Kongress, sowohl die Aufarbeitung der nationalsozialistischen Vergangenheit unter antifaschistischen Vorzeichen zu thematisieren als auch angesichts des beginnenden Kalten Krieges die Verantwortung der Schriftsteller für die deutsche Nation sowie die Sicherung des Friedens zu thematisieren. Folglich legte Becher zu Kongressbeginn die Linie der Kulturbundführung offen: Er sprach vom Friedens- und Einheitswillen des deutschen Volkes und forderte die Einheit einer deutschen antifaschistischen Literatur, die einen gesamtdeutsch-nationalen Beitrag für eine zukünftige gerechte Weltordnung zu leisten habe. ${ }^{230}$

Die eigentlichen Konflikte brachen am zweiten Tag der Veranstaltung auf und fanden ihren Höhepunkt am dritten, obwohl sie bereits in der Begrüßungsansprache des Vertreters der sowjetischen Militäradministration vorgezeichnet waren. Ganz im Sinne der neu festgelegten Propagandalinie von Schdanow und der Kominform-Führung sagte nämlich Oberstleutnant Alexander Dymschitz: »Der Kampf zwischen dem Neuen und dem Alten, zwischen Demokratie und Reaktion entwickelt sich in Ihrem Lande mit ungewöhnlicher Intensität und die Sprache des Schwertes erstarkt mit jedem

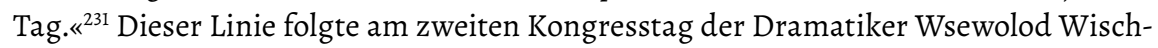

229 Vgl. hierzu grundsätzlich Ursula Reinhold/Dieter Schlenstedt/Horst Tannenberger (Hg.), Erster Deutscher Schriftstellerkongreß, 4.-8. Oktober 1947. Protokoll und Dokumente, Berlin 1997.

230 Hochgeschwender, Freiheit in der Offensive?, S. 140.

231 Zit. n. Reinhold/Schlenstedt/Tannenberger, Erster Deutscher Schriftstellerkongreß, S. 87. 
newski aus der sowjetischen Delegation, wobei die antiamerikanischen Töne seines antipazifistischen Plädoyers kaum überhörbar waren. Für ihn teilte sich die Welt in "zwei Teile«, wobei der eine Teil durch die »schwarze Reaktion, durch die Barbarei, durch die Ideologie des Menschenhasses und des Militarismus« vertreten wurde und der »andere Teil« durch die sogenannten Millionen einfacher Menschen, die »für die Demokratie kämpfen«; wie die »sowjetischen Schriftsteller «. ${ }^{232}$ Nachdem zwei weitere sowjetische Autoren, Valentin Katajew und Boris Gorbatow, auf den "antiamerikanischen Zug « aufsprangen, kam es am dritten Veranstaltungstag zum eigentlichen Eklat. Auslöser war der Auftritt des in letzter Minute ins Programm aufgenommenen amerikanischen Journalisten Melvin J. Lasky. Er war von Günther Birkenfeld nach dem Auftritt der sowjetischen Delegation in der Nacht zum 6. Oktober angesprochen wurden, der ihn zu einem Auftritt überredete. ${ }^{233}$ Birkenfeld, der am dritten Tag die Tagungsleitung innehatte, setzte Lasky auf die Rednerliste, ohne mit dem Kulturbundpräsidium Rücksprache gehalten zu haben. ${ }^{234}$

Lasky, der im Oktober des nächsten Jahres den Monat herausgab, war ehemaliger Kriegshistoriker des amerikanischen Heeres und schrieb zu diesem Zeitpunkt für die US-Zeitschrift New Leader und Partisan Review sowie den mit einer amerikanischen Lizenz ausgestatteten Berliner Tagesspiegel. Mit Blick auf die »totalitäre Kultur « des Nationalsozialismus und seine Verwüstungen am »deutschen Geist entwarf Lasky zunächst ein positives Bild der kulturellen und bürgerlichen Freiheiten und speziell des Rechtes auf uneingeschränkte Meinungsfreiheit in den USA, um ihm die durch Zensur und Unterdrückung gekennzeichnete Situation der Schriftsteller und Künstler in der Sowjetunion entgegenzuhalten. Sie lebten in dauernder Sorge, ob sie sich in Einklang mit der neuesten Parteidoktrin befänden, und riskierten demzufolge quasi über Nacht als >dekadente konterrevolutionäre Werkzeuge der Reaktion` abgestempelt zu werden. Zum Schluss erklärte sich Lasky angesichts der politischen Verfolgung der Schriftsteller Michail Soschtschenko und Anna Achmatowa in der Sowjetunion mit ihnen solidarisch und betonte unter Hervorhebung der "grundsätzlichen Prinzipien der kulturellen Freiheit«, dass die Rolle des Schriftstellers in jedem Gesellschaftssystem automatisch eine genuin oppositionelle ist. ${ }^{235}$

Nachdem die Rede Laskys für Tumult unter den Kongressteilnehmern sorgte, blieb es Walentin Katajew vorbehalten, in seiner direkten Erwiderung den Standpunkt der sowjetischen Delegation klipp und klar zum Ausdruck zu bringen. Wie Lasky richtete sich auch Katajew nicht in erster Linie an die Vertreter der anderen Großmacht, sondern an die deutschen Teilnehmer, um sie für die eigene Seite zu gewinnen. »Ich habe mich«, so Katajew zu Beginn, »mit der Rede des amerikanischen sogenannten Schriftstellers Lasky bekannt gemacht. Ich bin sehr froh, daß ich endlich einen lebendigen Kriegsbrandstifter zu Gesicht bekommen habe.«Im Verlauf seiner Rede, die vor allem durch den Versuch gekennzeichnet war, die Anklagen Laskys gegenüber der Sowjetunion als reine Lügen zu bezeichnen, scheute sich Katajew nicht, sie mit denen des

232 Zit. n. ebd., S. 245.

233 Hartmann/Eggeling, Sowjetische Präsenz im kulturellen Leben der SBZ und frühen DDR 1945-1953, S. 52.

234 Hochgeschwender, Freiheit in der Offensive?, S. 371.

235 Siehe die vollständige Lasky-Rede in: Reinhold/Schlenstedt/Tannenberger, Erster Deutscher Schriftstellerkongreß, S. 295-301. 
»verstorbene[n] Dr. Goebbels« zu vergleichen, der sich »derselben Mittel« bei seiner "Hetzerei gegen die Sowjetunion « bediente. ${ }^{236}$

Der zwischen der sowjetischen Delegation und Lasky im Oktober 1947 auf dem ersten gesamtdeutschen Schriftstellerkongress in Berlin ausgetragene Streit war ein Symptom für den ausbrechenden Kalten Krieg, der dann mit der Berlinkrise im darauf folgenden Jahr seinen ersten Höhepunkt fand. Der Kongress besiegelte zudem symbolisch die Teilung der deutschen Intellektuellen entlang der Frontlinien des Ost-West-Konfliktes. Der im Oktober in Berlin verabredete zweite gesamtdeutsche Schriftstellerkongress, der im Mai 1948 in Frankfurt a. M. stattfand, war bereits ausdrücklich durch den existenziellen Gegensatz zwischen den beiden Supermächten geprägt und ging ohne die ostdeutschen Autoren über die Bühne. ${ }^{237}$

Die Versuche der Sowjetunion durch den Kulturbund im Rahmen einer grundsätzlichen antifaschistisch-»demokratischen« Kulturpolitik in Deutschland, nationalneutralistische Kreise und friedensorientierte Linksintellektuelle für sich zu gewinnen, um die beiden miteinander verbundenen Ziele zu erreichen - die Spaltung der deutschen Nation zu verhindern und das eigene politische System in Deutschland einzuführen -, führte nicht nur zur Aufspaltung der deutschen Schriftsteller. Vor dem Hintergrund der »Zwei-Lager-Theorie« Schdanows und dem In-Gang-Kommen der antiamerikanischen Propaganda der Kominform - wie er zum Beispiel auf dem denkwürdigen ersten deutschen Schriftstellerkongress durch die sowjetische Delegation zum Ausdruck kam - waren die ideologischen und mithin die politischen Fronten geklärt. Aufseiten der amerikanischen Besatzungsbehörde setzten seit Oktober 1947 mit Blick auf die künftige Deutschlandpolitik vermehrt konkrete Planungen ein, die dieser Situation Rechnung trugen. Die Gründung der Zeitschrift Der Monat im Oktober 1948 war ein unmittelbares Resultat ebendieser Planungen.

\subsection{Die Gründung und die Funktion des Monat}

Im Rahmen seiner auf einer umfangreichen Quellenbasis beruhenden Studie Freiheit in der Offensive? Der Kongreß für kulturelle Freiheit und die Deutschen geht Hochgeschwender auch auf die facettenreichen Aspekte der Gründung und der Funktion der Zeitschrift Der Monat ein. ${ }^{238}$

Danach kündigte der amerikanische Militärgouverneur Clay angesichts der sich zuspitzenden politischen Situation in Deutschland auf einer Pressekonferenz am 28. Oktober $1947^{239}$ in Berlin die Operation »Talk Back« an, mit der unter genuin antikom-

236 Siehe die Replik von Valentin Katajew, in: Ebd., S. 336 f. (Zitate S. 336).

237 So Waltraud Wende-Hohenberger (Hg.), Der erste gesamtdeutsche Schriftstellerkongreß nach dem Zweiten Weltkrieg im Ostsektor Berlins vom 4. bis 8. Oktober 1947, Frankfurt a. M. u. a. 1988, S. XXVIII u. XXIX (Vorwort).

238 Siehe Hochgeschwender, Freiheit in der Offensive?, S. 139-203. Die nachfolgenden Ausführungen beziehen sich in erster Linie auf die Untersuchungsergebnisse dieser Studie, die neben Archivmaterial auch die zurzeit verfügbare Forschungsliteratur einbezieht. Es wird im Folgenden darauf verzichtet, die von ihm verwendeten Quellen jeweils im Einzelnen aufzuführen. Wenn inhaltliche Aspekte beleuchtet werden, die von ihm in seiner Studie nicht berücksichtigt wurden oder Präzisierungen vorgenommen werden, sind die jeweiligen Quellen aufgeführt.

239 So Mosberg, REeducation, S. 143. 